\title{
Broadband polarization beam splitter on a silicon nitride platform for O-band operation
}

\author{
Sylvain Guerber ${ }^{\mathbb{D}}$, Carlos Alonso-Ramos, Daniel Benedikovic, Elena Durán-Valdeiglesias, Xavier Le \\ Roux, Nathalie Vulliet, Eric Cassan, Delphine Marris-Morini, Charles Baudot, Frédéric Boeuf and \\ Laurent Vivien
}

\begin{abstract}
Wideband polarization beam splitters (PBS) are a key building block in polarization management circuits for wavelength division multiplexing (WDM) applications. A broadband PBS on a $300 \mathrm{~mm}$ silicon nitride (SiN) photonic platform is reported, based on phase-controlled directional coupler (DC). Unlike classical DC-based PBS made on silicon, the proposed configuration leverages the comparatively lower birefringence in SiN to yield wideband and efficient splitting. A semi-analytical approach, based on transfer matrix modelling and 3D-FDTD, has been used to design and optimize the structure for O-band operation. The proposed PBS has been experimentally demonstrated showing insertion loss (IL) lower than $0.6 \mathrm{~dB}$ and extinction ratio (ER) as high as $10 \mathrm{~dB}$ over 95 $\mathrm{nm}$ bandwidth for both polarizations. Furthermore, this simplified architecture eases cascading, allowing the demonstration of IL below $1.1 \mathrm{~dB}$ and ER larger than $24 \mathrm{~dB}$ over $85 \mathrm{~nm}$ wavelength range in a dual-stage configuration.
\end{abstract}

Index Terms-Optical polarization, Photonic integrated circuits, Silicon photonics, Silicon Nitride, Polarization beam splitter, Wavelength division multiplexing.

\section{INTRODUCTION}

I $\mathrm{N}$ recent years, emerging technologies from Cloud services (computing, working, streaming, gaming) together with Big Data are driving Internet traffic towards unprecedented density and speed levels [1]. A key point to this ramp up is that more than $75 \%$ of the global data traffic occurs inside datacenters [2], i.e. in a short distance range. Within this context, optical data communication links in the original telecom band (O-band) are spreading widely due to standardization. Silicon photonics, based on CMOS industrial manufacturing infrastructure, can fulfill those new requirements in terms of bandwidth and costs. However,

This work was supported in part by the European Research Council (ERC POPSTAR) under Grant 647342, in part by the European Commission H2020-ICT-27-2017 (COSMICC) under Grant 688516 and in part by the French Industry Ministry Nano2017 program.

S. Guerber is with the Centre de Nanoscience et Nanotechnologies (C2N), CNRS, Université Paris Sud, Université Paris Saclay, Orsay, 91405 FRANCE and with TR\&D STMicroelectronics SAS, Crolles, 38920 FRANCE (e-mail: sylvain.guerber@st.com).

C. Alonso-Ramos, D. Benedikovic, E. Durán-Valdeiglesias, X. Le Roux, E. Cassan, D. Marris-Morini, and Laurent Vivien are with Centre de Nanoscience et Nanotechnologies (C2N), CNRS, Université Paris Sud, Université Paris Saclay, Orsay, 91405 France.

N. Vulliet, C. Baudot and F. Boeuf are with TR\&D STMicroelectronics SAS, Crolles, 38920 FRANCE. silicon photonics circuits mostly rely on the high-indexcontrast $(\Delta \mathrm{n} \approx 2)$ silicon-on-insulator (SOI) platform, that suffers from high temperature sensitivity, tight fabrication tolerances, strong polarization dependence and large waveguide dispersion which in turn significantly constraints the devices performance. These limitations may be overcome by co-integration of silicon nitride $(\mathrm{SiN})$ [3], a CMOS compatible material. Indeed, $\mathrm{SiN}$ has a seven-fold lower thermo-optical coefficient compared with silicon [4]. Moreover, at the cost of a slightly larger footprint compared to SOI, SiN reduced index contrast $(\Delta \mathrm{n} \approx 0.5)$ leads to relaxed fabrication tolerances, efficient chip-fiber coupling as well as reduced wavelength-dependence of devices operation. Taking benefit of those properties, various applications have been demonstrated such as integrated spectrometers [5], quantum photonic circuits [6] and low power Kerr-comb generation [7].

Light polarization management has been recognized as a critical functionality in photonic chips over the years. Polarization splitting grating couplers (PSGC) [8], smartly implement this function together with chip-fiber coupling. Yet, their narrow bandwidth is incompatible with wavelength division multiplexing (WDM) applications. Another promising solution consists in using a broadband and polarization independent fiber-chip interface based on edge coupling $[9,10]$ together with a wideband polarization beam splitter (PBS). Compact and wideband PBS have been demonstrated in the SOI platform, based on highly birefringent bent directional couplers (DC) [11]. However, this approach is not well suited for the lower index contrast $\mathrm{SiN}$ platform. Wideband SOI PBS have also been shown [12], based on phase-controlled DC [13]. In this case, the large birefringence of SOI precludes simultaneously achieving both, proper coupling ratios for orthogonal polarizations and wideband operation. To circumvent this limitation, two wideband half splitters need to be cascaded, complicating device implementation. In this work, we propose and experimentally demonstrate a novel and flexible strategy that leverages the low birefringence of the $\mathrm{SiN}$ to implement wideband PBS with a single phase-controlled DC. The device is optimized to operate in the O-band, targeting WDM systems for datacom applications.

\section{DESIGN AND SIMULATION}

The PBS proposed here exploits the phase-controlled DC 
concept to yield wideband operation [13]. As shown on Fig. 1, the PBS comprises a phase-controlled DC, which can be divided into three parts: two symmetric coupling sections at each end, and a phase control region in between. According to this configuration, the wavelength-dependent behavior of the first symmetrical coupler section is compensated introducing a small phase shift prior to the second section.

The thickness of the SiN layer is $600 \mathrm{~nm}$, its refractive index is 1.92 at $1.3 \mu \mathrm{m}$ wavelength. In order to ensure single mode operation simultaneously for both orthogonal transverse electric (TE) and transverse magnetic (TM) polarizations, waveguide width $\left(\mathrm{W}_{\text {std }}\right)$ is fixed to $700 \mathrm{~nm}$. Coupling section gap $(\mathrm{G})$ is set to $600 \mathrm{~nm}$ as a tradeoff between footprint and fabrication sensitivity.

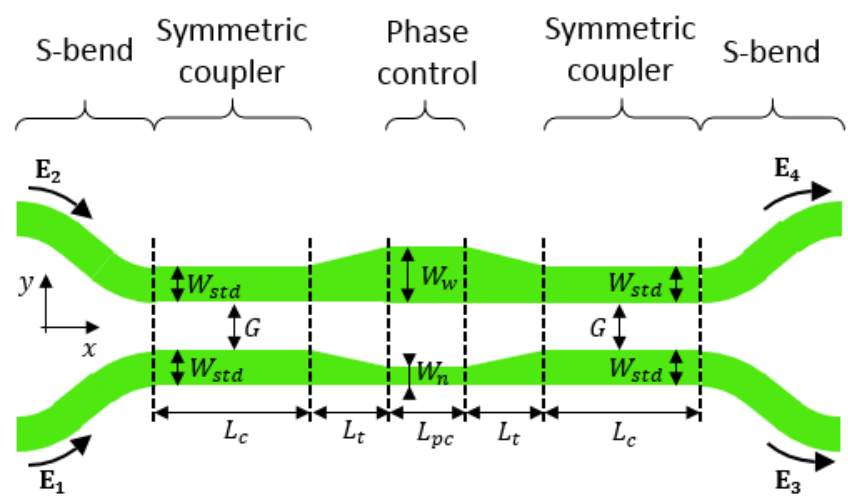

Fig. 1. In-plane (top) schematic view of the proposed polarization beam splitter.

Waveguide widths in the phase control section are respectively set to $850 \mathrm{~nm}$ for top arm $\left(\mathrm{W}_{\mathrm{w}}\right)$, limiting high order mode excitation, and $550 \mathrm{~nm}$ for bottom arm $\left(\mathrm{W}_{\mathrm{n}}\right)$, avoiding additional losses. Transition tapers of $6 \mu \mathrm{m}$ length $\left(\mathrm{L}_{\mathrm{t}}\right)$ are used to provide smooth mode transformation (low losses) while maintaining a compact footprint. S-bends are based on $40 \mu \mathrm{m}$ radius circular curves.

First, the device is analyzed using a semi-analytical transfer matrix modeling (TMM) approach [13]. Here, each particular section of the PBS is depicted by a transfer matrix as follows:

$$
\left[\begin{array}{c}
E_{3} \\
E_{4}
\end{array}\right]=C * P_{t} * P * P_{t} * C *\left[\begin{array}{c}
E_{1} \\
E_{2}
\end{array}\right]
$$

Input and output electric fields, $E_{1}$ to 4 , are as described in Fig. 1. Matrices $\mathrm{C}$ stand for symmetric coupler sections, whereas the transition tapers and the phase control region are described by Pt and P, respectively. Transfer matrices account for waveguide properties such as modal effective indices, losses and section length. Since no coupling is considered within the transition tapers and phase control sections, $\mathrm{P}_{\mathrm{t}}$ and $\mathrm{P}$ stand as diagonal matrices. In this modeling approach, the coupling occurring in the S-bend sections is not taken into account. Modal effective indices of each particular sections are calculated using a full-vectorial finite difference eigenmode (FDE) solver [14].

From this semi-analytical approach, two important parameters [13] are extracted:

$$
\eta_{\text {cross }}=\frac{\left|E_{4}\right|^{2}}{\left|E_{4}\right|^{2}+\left|E_{3}\right|^{2}}
$$

$$
\Delta \eta_{\text {cross }}=\left|\eta_{\text {cross }}(1.3)-\eta_{\text {cross }}(\lambda)\right|_{\text {max }}
$$

First one, as stated in (2), is the cross-coupling coefficient, $\eta$, which is defined as the normalized cross-power ratio between the two output arms. Second parameter, defined in (3), is the cross-coupling coefficient variation over $100 \mathrm{~nm}$ bandwidth, $\Delta \eta$, accounting for the device wavelength sensitivity. Both parameters have been computed for TE ( $\eta_{\mathrm{TE}}$, $\left.\Delta \eta_{\mathrm{TE}}\right)$ and TM $\left(\eta_{\mathrm{TM}}, \Delta \eta_{\mathrm{TM}}\right)$ polarizations. To perform the optimization of the splitting efficiency and the bandwidth of the PBS, we have defined two figures of merit, $\eta_{P B S}$ and $\triangle \eta_{P B S}$, that relate aforementioned parameters as:

$$
\begin{gathered}
\eta_{P B S}=\left|\eta_{T E}-\eta_{T M}\right| \\
\Delta \eta_{P B S}=\frac{\Delta \eta_{T E}+\Delta \eta_{T M}}{2}
\end{gathered}
$$

$\eta_{P B S}$, as stated in (4), represents the PBS splitting efficiency, with the best splitting performance obtained for $\eta_{P B S}=1$. Whereas $\Delta \eta_{P B S}$, defined in (5), stands for the PBS wavelength sensitivity; the lower $\Delta \eta_{P B S}$, the broader bandwidth. Coupler characteristics, $\eta_{\mathrm{PBS}}$ and $\Delta \eta_{\mathrm{PBS}}$, are then tuned by adjusting the length of symmetrical couplers $\mathrm{L}_{c}$, and the length of phase control section $\mathrm{L}_{\mathrm{pc}}$. The results are respectively reported in Fig. 2(a) and 2(b).
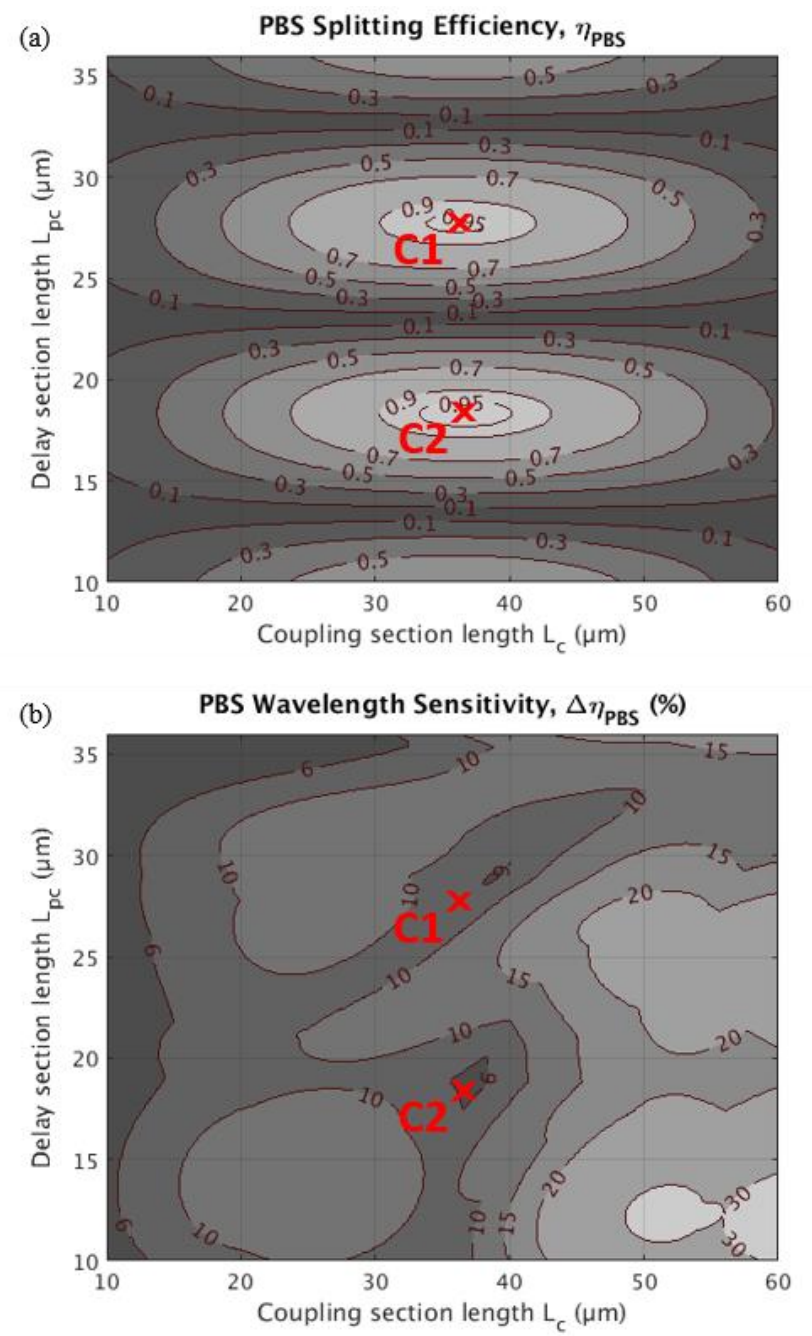

Fig. 2. Filled contour maps of PBS splitting efficiency, $\eta$ PBS (a), and wavelength sensitivity, $\triangle \eta \mathrm{PBS}$ (b), as function of Lc and Lpc. Red crosses denoted $\mathrm{C} 1$ and $\mathrm{C} 2$ shows two interesting (Lc, Lpc) couples for PBS design. 
Fig. 2(a) reveals two $\left(\mathrm{L}_{\mathrm{c}}, \mathrm{L}_{\mathrm{pc}}\right)$ couples, denoted by $\mathrm{C} 1$ and $\mathrm{C} 2$, that achieve efficient polarization splitting. Those specific configurations occur thanks to the slightly birefringent response of the SiN waveguides. Indeed, different phase shift and coupling strength are introduced for TE and TM polarizations allowing the phase-controlled DC to act as a PBS. As shown, for $\mathrm{L}_{\mathrm{c}}=36.2 \mu \mathrm{m}$ and $\mathrm{L}_{\mathrm{pc}}=27.8 \mu \mathrm{m}(\mathrm{C} 1)$, $\eta_{\text {PBS }}=0.96$ while for $\mathrm{L}_{\mathrm{c}}=36.6 \mu \mathrm{m}$ and $\mathrm{L}_{\mathrm{pc}}=18.4 \mu \mathrm{m}(\mathrm{C} 2)$, $\eta_{\mathrm{PBS}}=0.97$. Fig. 2(b) shows that $\Delta \eta_{\mathrm{PBS}}$ is slightly higher for $\mathrm{C} 1\left(\Delta \eta_{\mathrm{PBS}} \approx 7.2 \%\right)$ compared to $\mathrm{C} 2\left(\Delta \eta_{\mathrm{PBS}} \approx 5.5 \%\right)$. Consequently, with a higher splitting efficiency and a lower wavelength sensitivity, the second $\left(\mathrm{L}_{\mathrm{c}}, \mathrm{L}_{\mathrm{pc}}\right)$ couple $(\mathrm{C} 2)$ is selected for the final device design.

The selected design is fine-tuned using three-dimensional Finite Difference Time Domain (3D-FDTD) [13] simulations. Considering the S-bend contributions, the optimal values for the coupling and phase control regions are determined to be $\mathrm{L}_{\mathrm{c}}$ $=30 \mu \mathrm{m}$ and $\mathrm{L}_{\mathrm{pc}}=18 \mu \mathrm{m}$. Fig. 3 shows the propagation of both TE and TM fields for the optimized design at a nominal wavelength of $1300 \mathrm{~nm}$.
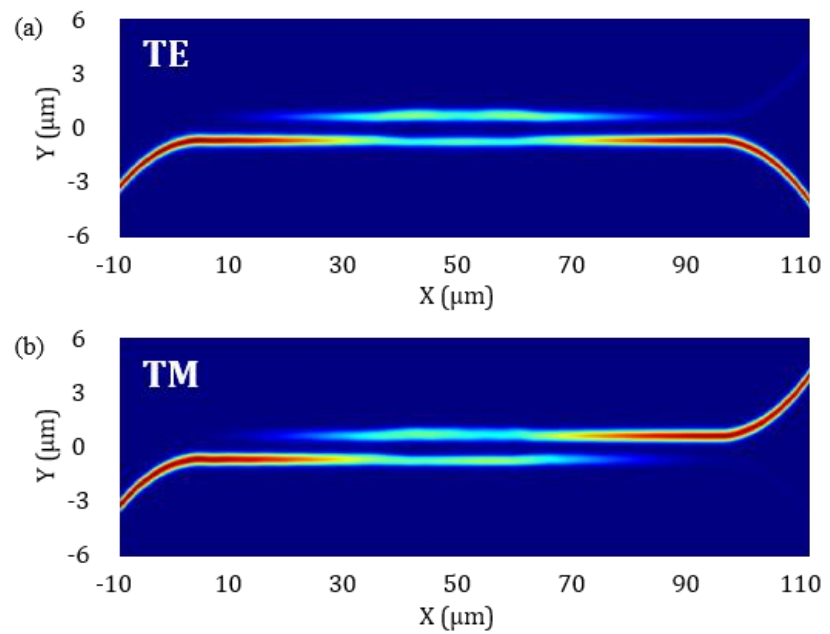

Fig. 3. Optical field distribution in the PBS from 3D-FDTD for (a) TE and (b) TM polarizations at $\lambda=1.3 \mu \mathrm{m}$.

\section{FABRICATION AND CHARACTERIZATION}

Devices have been fabricated on the $300 \mathrm{~mm}$ silicon photonics R\&D platform DAPHNE in ST Crolles (France) [3, 15]. We used silicon bulk wafers with $1.4 \mu \mathrm{m}$-thick silicon dioxide $\left(\mathrm{SiO}_{2}\right)$ layer as a buried oxide. A $600 \mathrm{~nm}$-thick $\mathrm{SiN}$ layer is then deposited at low temperature via plasma enhanced chemical vapor deposition (PECVD). SiN patterning is performed using $248 \mathrm{~nm}$ deep-ultraviolet (deep-UV) optical photolithography, followed by dry etching process. An encapsulation layer of a $1.5 \mu \mathrm{m}$ thick silicon dioxide is finally deposited to complete the fabrication process.

Individual dies have been singled out to perform optical characterization in an edge coupling scheme. High precision stealth dicing has been carried out to achieve a good lateral surface uniformity. Consequently, reliable edge coupling is obtained leading to repeatable coupling efficiency for all waveguides included within the sample.
Devices optical testing has been performed over a wavelength range from $1260 \mathrm{~nm}$ to $1355 \mathrm{~nm}$. Light is injected in the bottom arm (E1) and collected for both outputs (E3 and E4) and for both TE and TM polarizations. The control of light polarization state has been performed using standard fiber paddle polarization controller together with calcite-made polarizers in order to proper reject the unwanted polarization states on a large wavelength range. The measured spectral responses are normalized with a straight waveguide transmission in the respective polarization state. Device performance is then evaluated from the insertion losses (IL) and the extinction ratio (ER), which is defined by the power difference between both polarizations in a given output arm.

Fig. 4(a) shows the experimental spectral response of a single PBS. Insertion losses lower than $0.6 \mathrm{~dB}$ and ER above $10 \mathrm{~dB}$ are achieved for both TE and TM polarizations, over the $95 \mathrm{~nm}$ wavelength range. This confirms the simulated broadband behavior of our devices.
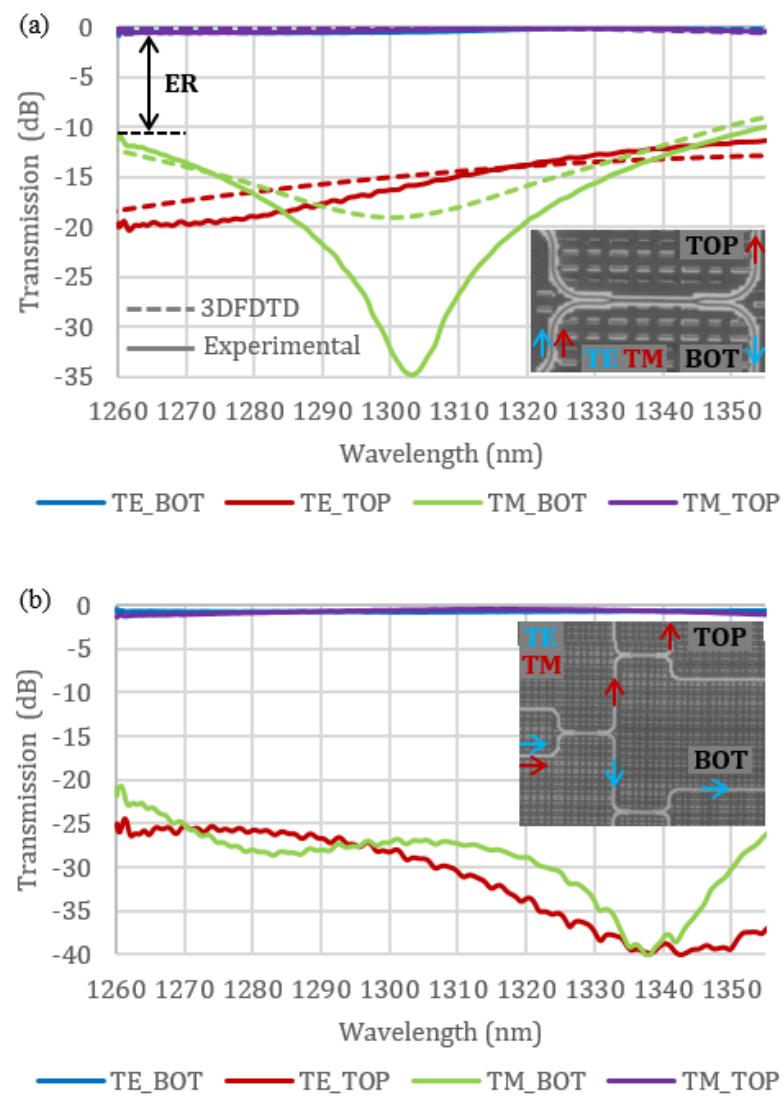

Fig. 4. Simulated and experimental spectral response for (a) single PBS and (b) cascaded PBS. Insets shows top view of devices from optical microscope with polarization response.

However, some applications like coherent communication systems [16] typically require comparatively higher ER levels. Therefore, a cascaded structure comprising three PBS has been realized in order to achieve stronger polarization filtering. This circuit configuration is shown in the inset of Fig. 4(b). As a result, two times improvement in respect to ER is achieved, reaching more than $20 \mathrm{~dB}$ for both polarizations 
over $95 \mathrm{~nm}$ bandwidth while keeping the IL below $1.3 \mathrm{~dB}$. An ER level of $24 \mathrm{~dB}$ is even achieved over $85 \mathrm{~nm}$ bandwidth with IL lower than $1.1 \mathrm{~dB}$.

Comparison with state of the art is presented in Table I. The reported devices exhibit comparable performances with $\mathrm{C}$ and L-band SOI-based PBS in terms of insertion losses, extinction ratio, and bandwidth. It is also worth noting that the wideband PBS's realized in this work are the first experimental demonstration of such photonic components on a $\mathrm{SiN}$ platform, completely fabricated in an industrial environment. These results pave the way towards implementation of dualpolarization applications, such as polarization diversity or coherent communications, in a CMOS-compatible SiN material system, taking advantage of the platform stability related to environment and fabrication.

TABLE I

COMPARISON WITH STATE OF THE ART

\begin{tabular}{cccccc}
\hline \hline Ref. & $\begin{array}{c}\text { IL }(\mathrm{dB}) / \\
\mathrm{BW}(\mathrm{nm})\end{array}$ & $\begin{array}{c}\text { ER } \\
(\mathrm{dB})\end{array}$ & $\begin{array}{c}\text { Wavelength } \\
(\mathrm{nm})\end{array}$ & $\begin{array}{c}\text { Footprint } \\
(\mathrm{L} \times 1) \mu \mathrm{m}\end{array}$ & Mat. \\
\hline$[12]$ & $>\mathrm{NA} / 125$ & $>20$ & $1477-1602$ & $97.4 \times 5.5$ & $\mathrm{SOI}$ \\
{$[17]$} & $>1 / 35$ & $>17$ & $1530-1565$ & $4.8 \times 1.6$ & $\mathrm{SOI} /$ \\
& & & & & $\mathrm{SiN}$ \\
{$[18]$} & $>\sim 0.8 / 95$ & $>25$ & $1500-1595$ & $20 \times 6.9$ & $\mathrm{SOI}$ \\
{$[19]$} & $>1.3 / 100$ & $>9.9$ & $1470-1570$ & $5.8 \times \mathrm{NA}$ & $\mathrm{SOI}$ \\
{$[20]$} & $>2.2 / 55$ & $>10$ & $1525-1580$ & $132.6 \times 4.2$ & $\mathrm{SOI}$ \\
{$[21]$} & $>3.4 / 150$ & $>10$ & $1350-1500$ & $1400 \times 1.8$ & $\mathrm{SOI}$ \\
$\mathrm{WK} 1$ & $>0.6 / 95$ & $>10$ & $1260-1355$ & $136 \times 8.7$ & $\mathrm{SiN}$ \\
$\mathrm{WK} 2$ & $>1.3 / 95$ & $>20$ & $1260-1355$ & - & $\mathrm{SiN}$ \\
$\mathrm{WK} 3$ & $>1.1 / 85$ & $>24$ & $1270-1355$ & - & $\mathrm{SiN}$ \\
\hline \hline
\end{tabular}

WK1: This work, design of record; WK2: This work, cascaded version; WK3: This work, cascaded version, high ER.

\section{CONCLUSION}

In conclusion, a novel architecture of broadband polarization beam splitter is proposed and realized using a 300 $\mathrm{mm} \mathrm{SiN} \mathrm{platform}$. The devices were seamlessly fabricated in a single-etch step process by conventional 248-nm deep-UV optical lithography, typically used by industrially-driven photonic applications. Optical characterizations demonstrated insertion loss lower than $0.6 \mathrm{~dB}$ and extinction ratio larger than $10 \mathrm{~dB}$ for both TE and TM polarizations, covering the full O-band. Furthermore, a cascaded architecture achieves insertion losses below $1.3 \mathrm{~dB}$, with an extinction ratio larger than $20 \mathrm{~dB}$ on the same wavelength range.

\section{REFERENCES}

[1] CISCO, "Global Cloud Index 2015-2020," [Online]. Available: https://www.cisco.com/

[2] CISCO, "Global Cloud Index 2015-2020: Forecast and Methodology," [Online]. Available: https://www.cisco.com/

[3] C. Baudot et al., "Developments in $300 \mathrm{~mm}$ silicon photonics using traditional CMOS fabrication methods and materials," in Proc. IEEE-IEDM, San Francisco, CA, USA, 2017, pp. 34.3.1 - 34.3.4.

[4] Z. Zhang, M. Yako, K. Ju, N. Kawai and K. Wada, "A Silicon Nitride Platform by PVD for Dense Wavelength Division Multiplexing on Chip," in Proc. IEEE-GFP, Paris, France, 2014, pp.193-194.
[5] X. Nie, E. Ryckeboer, G. Roelkens, and R. Baets, "CMOScompatible broadband co-propagative stationary Fourier transform spectrometer integrated on a silicon nitride photonics platform," Opt. Exp., vol. 25, no. 8, pp. 409-418, 2017.

[6] M. Poot, C. Schuck, X.-s. Ma, X. Guo, and H. X. Tang, "Design and characterization of integrated components for SiN photonic quantum circuits," Opt. Exp., vol.24, no. 7, pp. 6843-6860, 2016.

[7] Y. Xuan, Y. Liu, L. T. Varghese, A. J. Metcalf, X. Xue, P.H. Wang, K. Han, J. A. Jaramillo-Villegas, A. A. Noman, C. Wang, S. Kim, M. Teng, Y. J. Lee, B. Niu, L. Fan, J. Wang, D. E. Leaird, A. M. Weiner, and M. Qi, "High-Q silicon nitride microresonators exhibiting low-power frequency comb initiation," Optica, vol. 3, no. 11, pp. 11711180, 2016.

[8] W. Bogaerts, D. Taillaert, P. Dumon, D. Van Thourhout, and R. Baets, "A polarization-diversity wavelength duplexer circuit in silicon-on-insulator photonic wires," Opt. Exp., vol. 15, no. 4, pp. 1567-1578, 2007.

[9] K. Kasaya, O. Mitomi, M. Naganuma, Y. Kondo, and Y. Noguchi, "A simple laterally tapered waveguide for lowloss coupling to single-mode fibers," IEEE Photon. Technol. Lett., vol. 5, no. 1, pp 345-347, 1993.

[10] T. Barwicz, A. Janta-Polczynski, M. Khater, Y. Thibodeau, R. Leidy, J. Maling, S. Martel, S. Engelmann, J. S. Orcutt, P. Fortier, and W. M. J. Green, "An o-band metamaterial converter interfacing standard optical fibers to silicon nanophotonic waveguides," in Proc. OFC, Los Angeles, CA, USA, 2015, pp. 1-3.

[11] D. Dai and J. E. Bowers, "Novel ultra-short and ultrabroadband polarization beam splitter based on a bent directional coupler," Opt. Exp., vol. 19, no. 19, pp. 1861418620,2011

[12] Z. Lu, Y. Wang, F. Zhang, N. A. F. Jaeger, and L. Chrostowski, "Wideband silicon photonic polarization beamsplitter based on point-symmetric cascaded broadband couplers," Opt. Exp., vol. 23, no. 23, pp. 29413-29422 , 2015.

[13] Z. Lu, H. Yun, Y. Wang, Z. Chen, F. Zhang, N. A. F. Jaeger, and L. Chrostowski, "Broadband silicon photonic directional coupler using asymmetric-waveguide based phase control," Opt. Exp., vol. 23, no. 3, pp. 3795-3808, 2015.

[14] Lumerical Solutions, Inc., [Online]. Available: https://www.lumerical.com/tcad-products/

[15] S. Guerber et al., "Integrated SiN on SOI dual photonic devices for advanced datacom solutions," in Proc. SPIE 10686, Strasbourg, France, 2018.

[16] S. J. Savory, "Digital coherent optical receivers: algorithms and subsystems," IEEE J. Sel. Top. Quantum Electronics, vol. 16, no. 5, pp. 1164-1179, 2010.

[17] X. Sun, J. Stewart Aitchison, and M. Mojahedi, "Realization of an ultra-compact polarization beam splitter using asymmetric MMI based on silicon nitride / silicon-oninsulator platform," Opt. Exp., vol. 25, no. 7, pp. 8296-8305 , 2017.

[18] H. Wu, Y. Tan, and D. Dai, "Ultra-broadband highperformance polarizing beam splitter on silicon," Opt. Exp., vol. 25, no. 6, pp. 6069-6075, 2017.

[19] F. Zhang, H. Yun, Y. Wang, Z. Lu, L. Chrostowski, and N. A. F. Jaeger, "Compact broadband polarization beam splitter using a symmetric directional coupler with sinusoidal bends," Opt. Lett., vol. 42, no. 2, pp. 235-238, 2017.

[20] M. Yin, W. Yang, Y. Li, X. Wang, and H. Li, "CMOScompatible and fabrication-tolerant MMI-based polarization beam splitter," Opt. Commun., vol. 335, pp. 48-52, 2015.

[21] Z. Su, E. Timurdogan, E. S. Hosseini, J. Sun, G. Leake, D. D. Coolbaugh, and M. R. Watts, "Four-port integrated polarizing beam splitter," Opt. Lett., vol. 39, no. 4, pp. 965968, 2014. 